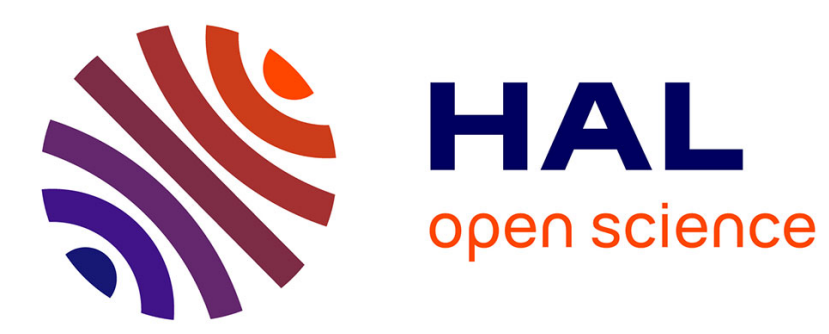

\title{
Assessing and Minimizing the Impact of OCR Quality on Named Entity Recognition
}

Ahmed Hamdi, Axel Jean-Caurant, Nicolas Sidère, Mickaël Coustaty, Antoine

Doucet

\section{- To cite this version:}

Ahmed Hamdi, Axel Jean-Caurant, Nicolas Sidère, Mickaël Coustaty, Antoine Doucet. Assessing and Minimizing the Impact of OCR Quality on Named Entity Recognition. Digital Libraries for Open Knowledge 24th International Conference on Theory and Practice of Digital Libraries, TPDL 2020, Lyon, France, August 25-27, 2020, Proceedings, pp.87-101, 2020, 10.1007/978-3-030-54956-5_7 . hal03026931

\section{HAL Id: hal-03026931 \\ https://hal.science/hal-03026931}

Submitted on 26 Nov 2020

HAL is a multi-disciplinary open access archive for the deposit and dissemination of scientific research documents, whether they are published or not. The documents may come from teaching and research institutions in France or abroad, or from public or private research centers.
L'archive ouverte pluridisciplinaire HAL, est destinée au dépôt et à la diffusion de documents scientifiques de niveau recherche, publiés ou non, émanant des établissements d'enseignement et de recherche français ou étrangers, des laboratoires publics ou privés. 


\title{
Assessing and Minimizing the Impact of OCR Quality on Named Entity Recognition
}

\author{
Ahmed Hamdi, Axel Jean-Caurant, Nicolas Sidere, Mickaël Coustaty, Antoine \\ Doucet \\ University of La Rochelle, France \\ \{firstname.lastname\}@univ-lr.fr
}

\begin{abstract}
The accessibility to digitized documents in digital libraries is greatly affected by the quality of document indexing. Among the most relevant information to index, named entities are one of the main entry points used to search and retrieve digital documents. However, most digitized documents are indexed through their OCRed version and OCR errors hinder their accessibility. This paper aims to quantitatively estimate the impact of OCR quality on the performance of named entity recognition (NER). We tested state-of-the-art NER techniques over several evaluation benchmarks, and experimented with various levels and types of OCR noise so as to estimate the impact of OCR noise on NER performance. To the best of our knowledge, no other research work has systematically studied the impact of OCR on named entity recognition over data sets in multiple languages. The final outcome of this study is an evaluation over historical newspaper data provided by the national library of Finland, resulting in a large increase over the best-known results to this day.
\end{abstract}

Keywords: Digitized documents $\cdot$ Indexing $\cdot$ OCR $\cdot$ Named entity recognition.

\section{Introduction}

Substantial amounts of printed documents are digitized and archived as images in digital libraries. This is notably the case of historical documents, which require an Optical Character Recognition (OCR) step for their textual content to be accessible. Unfortunately, while the performance of OCR systems has greatly improved, it remains imperfect. In addition, a great deal of documents were digitized in a time where storing high-quality images was costly. Such documents cannot readily benefit from improvements in OCR quality. Several studies understandably suggest that the performance of natural language processing tools is harmed by the use of text resulting from an OCR process [17]. This naturally makes document access more difficult since for instance simple keyword search will for instance not match a query with corresponding the words if they suffered from OCR errors. The quality of the text generated using OCR engines depends on the algorithms used in OCR, on the setting parameters of the scanner used to 
digitize documents, on the quality of the original image and on the nature of the document. For instance, text generated from recent and historical newspapers or normal and damaged manuscripts do not usually have the same quality. Even though a reasonable amount of OCR errors is known to have a relatively low impact on the readability of documents, the errors will be indexed as they are by search engines and other NLP tools. Subsequently, if some words are incorrectly recognized by the OCR process, they will be indexed with their errors. This causes a chain reaction for tools developed to analyze the resulting content.

A study has shown that named entities (NEs) are the first point of entry for users in a search system [10]. As an illustration, it has been observed that 4 out of 5 user queries on the Gallica digital library ${ }^{1}$ contain at least one named entity [2]. For this reason, their quality is far more critical than that of most other words in OCRed documents. In order to improve the satisfaction of users' information needs, it is thus necessary to ensure their quality.

Named entity recognition (NER) is a task that emerged in the middle of the $90 s$ [12]. It aims to locate and categorize important concepts of a given text into a set of predefined classes. Three main labels are commonly used: persons, locations and organizations [21]. NER techniques can be gathered in two groups: rule-based and machine learning methods. For rule-based methods, the rules are mainly defined manually. They are related to linguistic descriptions, trigger words and lexica of proper names. These rules use patterns and regular expressions in order to locate and classify named entities. Machine learning approaches, on the other hand, aim to extract rules automatically based on learning systems trained on large corpora. Rule-based methods are clearly affected by OCR errors and are not able to deal with the degradation generated by the OCR. On the other hand, machine learning methods present a sufficient flexibility to be automatically adapted to process noisy texts. More recently, neural networks have been shown to outperform other supervised algorithms for NER. The first deep neural network based learning system has been developed in 2011 [4]. It reached very competitive results for NER in comparison to previous machine learning approaches. Therefore, many NER systems using neural networks have been proposed and have shown their abilities to outperform all previous systems [24]. We present in this paper a comparative study of well-performing NER methods. We have chosen, in this work, to use four majors systems available: the well-known NER tool using Conditional Random Fields CoreNLP [8] and three neural network systems BLSTM-CRF [16], BLSTM-CNN [3] and BLSTM-CNNCRF [19]. The reason being that processing degraded texts using rule based systems require substantial manual efforts to face all typical OCR degradations, unlike machine learning systems which are able to automatically overcome OCR degradations. Furthermore, most of rule-based systems are domain-specific or language-dependant and can not be easily extended to other domains or other languages [9]. Our goal is to evaluate the impact of OCR error on NER accuracy when dealing with noisy texts, which is strongly related to indexing processes of documents in digital libraries. To the best of our knowledge, no other research

\footnotetext{
${ }^{1}$ Gallica is the digital portal of the National Library of France.
} 
work has systematically studied the impact of OCR on named entity recognition over data sets in multiple languages.

In order to assess our work, we used three publicly available data sets which cover three languages (English, Dutch and Spanish). Given the lack of OCRed annotated data aligned with its ground truth, we have simulated test data by adding typical textual degradation given by an OCR engine. These data have been obtained by automatically adding many levels of degradation in those corpora. More specifically, we spread four types of common OCR degradation in the original clean text. As OCR error depends on the quality and the parameters of the digitization process, we also simulated typical scanning noises at two different levels: rarely and reasonably frequently. We finally aligned clean and OCRed data in order to be able to use the same annotation data. Running NER systems through progressively noisy data allows us to estimate the curve of NER F-score results relatively to OCR error rates. Results over our simulated OCRed resource will show a general consistency with a real OCRed dataset extracted from Finnish historical newspaper provided by the national library of Finland.

The rest of the paper is organized as follows : Section 2 presents related work studying the impact of OCR. Section 3 consists in an overview of the data sets, followed by outlines of NER results over clean and OCRed texts in Section 4 . Section 5 reports our experiments with real data and Section 6 concludes the paper.

\section{Related work}

Despite decades of research, the output of OCR systems remains imperfect, especially when the analogical document is old, damaged or poorly digitized. OCR systems lie in the beginning of the digitalization pipeline and OCR errors tend to have a cumulative impact over the subsequent steps. For this reason, researchers have studied the impact of processing text data from noisy sources in order to understand the effects of OCR on text analysis tools.

Much research has been devoted in natural language processing (NLP) to process noisy data.Lopresti Daniel [17] for instance considered a text analysis pipeline consisting of sentence boundary detection, followed by tokenization and POS tagging. They reported that among the errors generated by the OCR process, insertion errors were worse than character deletion errors on the sentence boundaries task, while OCR substitution errors were more impactful on POS tagging. The effects of noisy texts have been evaluated also on other NLP tasks such as document summarization [14] and machine translation [33].

Many other works focused on information retrieval from noisy data [5]. Chiron et al. [2] proposed a method to estimate the impact of OCR errors on the use of digital libraries. They built an OCR error model using a large corpus of OCRed documents aligned with their corresponding ground truth. Their model allowed the estimation of the risk that a user's query might fail to match with the targeted documents. Taghva et al. [30] showed that moderate OCR error rates have not desperate impact on the effectiveness of classical information re- 
trieval measures. Other studies focused on the impact of OCR errors on the classification of pathology reports for cancer notification [34]. They concluded that OCR errors even with modest rates are not imperceptible for extraction cancer notification items.

For NER, several works have been done to extract NEs from diverse text types such as outputs of Automatic Speech Recognition (ASR) systems [7]informal SMS and noisy social network posts [27]. Palmerand Ostendorf [22] for example described an approach for improving named entity extraction from ASR systems outputs by explicitly modeling errors through the use of confidence scores. In a similar setting, Miller et al. [20] have studied the performance of named entity extraction under a variety of spoken and OCRed data. They trained the IdentiFinder system [1] on both clean and noisy input material, performance degraded linearly as a function of word error rates. They concluded that results may lose about 8 points of $\mathrm{F}$-score with only $15 \%$ of word error rate. Rodriquez et al. [28] reported that manual correction of OCR output have not a very observable improvement on NER results. In [26], Riedl et al. presented a complete framework for named entity recognition for both contemporary and historical German using transfer learning technique. They achieved state-of-the-art performance for historical datasets with less samples that contains noise.

In this paper, similarly to [28] and [20], we propose to study the evolution of the performance of named entity recognition systems over noisy OCR data. Unlike them we use more sophisticated NER systems relying on the most recent neural networks models. We also use larger corpora covering four languages, thanks to a technique that allows us to synthesize and test different types and levels of noise. They contain different types of degradation that correspond to the results of long storage and the impact of digitization processes. We defined two levels of degradation for each type in order to obtain a clearer view on OCR errors and their impact on the task of named entity recognition.

\section{Dataset overview}

To the best of our knowledge, no publicly available corpus has been found with named-entity annotations on both clean and noisy texts recognition at the same time. In addition, there are corpora where text produced by an OCR process is aligned with the original text but NEs are not annotated. For this reason, we have taken advantage of three available corpora, with annotations on NEs, and simulated from them several OCRed versions with variable OCR error rates. We used the public corpora (CoNLL-02 and CoNLL-03) dealing with named entities and covering three languages: English [31], Spanish and Dutch [6]. English data consist of Reuters news stories between August 1996 and August 1997. Spanish corpus is a collection of news wire articles made available by the Spanish EFE News Agency while Dutch corpus consists of four editions of the Belgian newspaper "De Morgen". Those datasets are split into three subsets: a training set, a test set and a development set. The latter has been built in order to tune parameters of learning methods. 
All data files contain a single word per line with its associated named entity tag. Part-of-speech tags have been automatically generated and provided with words. Chunk tags have been additionally provided for English data only. Table 1 outlines details about each dataset used in this work.

\begin{tabular}{|c|c|c|c|c|c|c|}
\hline & \multirow[t]{2}{*}{ sentences } & \multicolumn{2}{|c|}{ words } & \multicolumn{2}{|c|}{ named entities } \\
\hline & & & tokens & terms & tokens & terms \\
\hline \multirow{3}{*}{ Spanish } & Train & 8,323 & 264,715 & 26,099 & 32,795 & 6,821 \\
\hline & Dev & 1,915 & 52,923 & 9,646 & 7,567 & 2,377 \\
\hline & Test & 1,517 & 51,533 & 9,086 & 6,178 & 1,974 \\
\hline \multirow{3}{*}{ English } & Train & 14,987 & 204,567 & 23,624 & 29,450 & 6,955 \\
\hline & Dev & 3,466 & 51,578 & 9,967 & 7,335 & 2,735 \\
\hline & Test & 3,684 & 46,666 & 9,489 & 7,194 & 2,384 \\
\hline \multirow{3}{*}{ Dutch } & Train & 15,806 & 202,932 & 27,805 & 14,555 & 4,332 \\
\hline & Dev & 2,895 & 37,762 & 8,151 & 2,751 & 1,033 \\
\hline & Test & 5,195 & 68,995 & 11,803 & 4,170 & 1,567 \\
\hline
\end{tabular}

Table 1. CoNLL-02 and CoNLL-03 data sets

The annotation of named entities follows the IOB-scheme (Inside, Outside, Beginning) where every token is labeled as B-label if the token is the beginning of a named entity, I-label if it is inside but not the first token within the named entity, or O otherwise [25]. Four classes have been used to label NEs:

- PER: individual, group, indeterminate

- LOC: address, country, region, continent, GPE, nation, state or province

- ORG: commercial, educational, entertainment, government, media, medicalscience, non-governmental, religious, sports;

- MISC: all named entities not belonging to the other three classes.

From test data, we simulated several OCRed versions. To do so, we first extracted raw texts from test sets and converted them into images. These images have been contaminated by adding some common noises when using a scanner. We further extract OCRed data using tesseract open source OCR engine v$3.04 .01^{2}$ which provides a language package covering many languages among them English, Dutch and Spanish. Consequently to the image noise insertions, OCRed data contains degradations. Original and noisy texts are finally aligned and annotations of the original corpus have been projected back on the noisy version. Figure 1 describes the main steps to simulate noisy corpora. We assume that the target text is similar to the indexed text in digital libraries.

\footnotetext{
${ }^{2}$ https://github.com/tesseract-ocr/
} 
A. Hamdi et al.

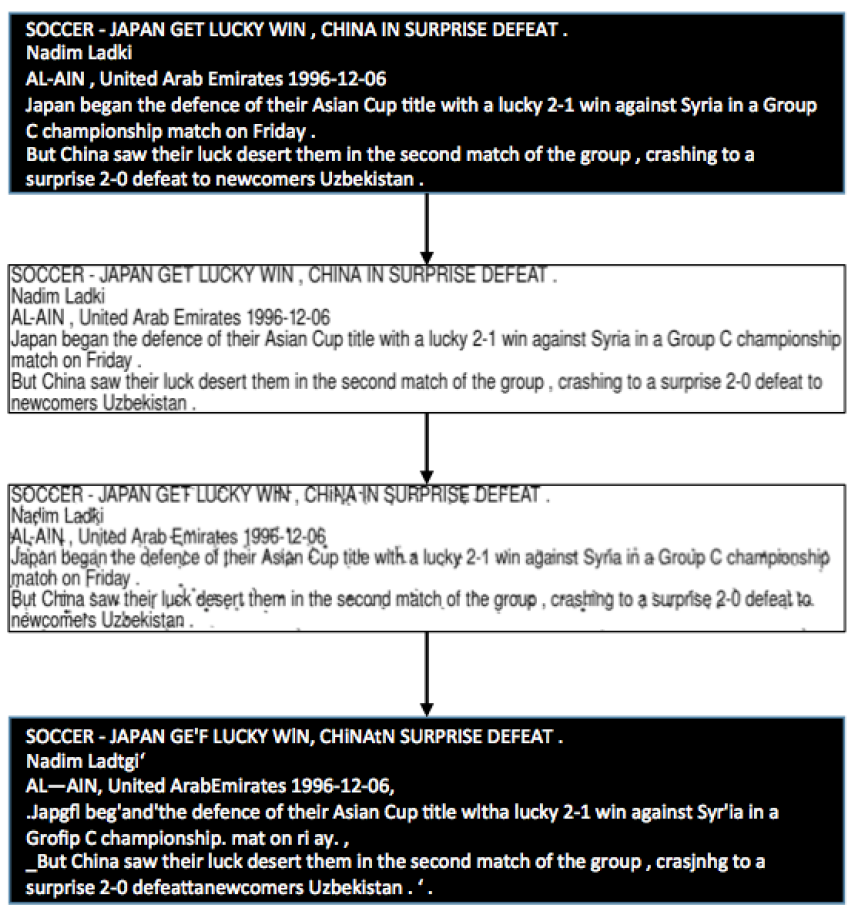

Fig. 1. Simulation of OCRed copora

In order to contaminate images, we have used the DocCreator tool ${ }^{3}$ developed by Journet et al.[15]. The tool provides many options to add degradation to document images such as blurring, ink degradation and adding phantom character. In this work, we applied four types of degradation related to storage conditions or poor quality of printing materials that may be present on digital libraries material:

- character degradation simulates degradation due to the age of the document or the use of a scanner incorrectly set. It consists in adding small ink spots on characters and can induce the erasure of characters.

- phantom degradation simulates degradation in worn documents. Following successive uses, some characters can be progressively eroded. The digitization process generates phantom ink around characters.

- bleed-through simulates verso ink seeping through recto side. This degradation appears only with double-sided pages.

- blurring simulates a blurring effect, as can be encountered during a typical digitization process with focus issue.

\footnotetext{
${ }^{3}$ http://doc-creator.labri.fr/
} 
For each type of noise, we defined two levels of degradation: LEV-1 where noises are applied rarely and LEV-2 where degradation are reasonably more frequent. These levels allow generating noisy texts with an OCR error rate close to real cases [13]. These degradation allowed building eight versions for each test corpus. We additionally defined two versions that we call respectively LEV-0 and LEV-MIX. The LEV-0 version is re-OCRred version of original images with no degradation added while the LEV-MIX version is the result of combining all the LEV-1 degradation types ${ }^{4}$. The LEV-0 degradation aims to evaluate the OCR engine through sharp images whereas the goal of using the LEV-MIX degradation is to be more similar to real-world documents. Degraded documents in digital libraries contains almost many OCR degradations simultaneously.

Following the text extraction by the OCR, noisy text have been aligned to its original version using the tool RETAS [32]. This tool allows us to make this correspondence.An example of alignment made between the ground truth and its OCRed version is shown in Figure 2. This alignment reflects the various errors made by the OCR engine. The difference between the two texts are denoted by the presence of the character '@'. Each '@' in the ground truth indicates the insertion of one character by the OCR while '@' in the noisy text indicates that one character has been deleted from the original text.

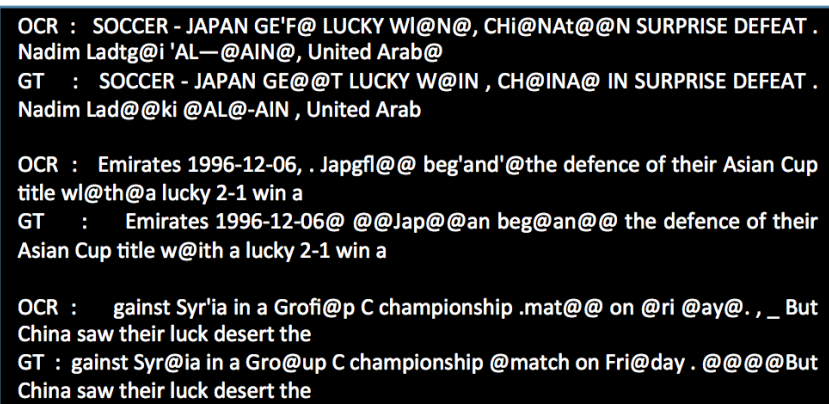

Fig. 2. Original and noisy texts alignment

In order to evaluate the OCR quality we used two measures: the Character Error Rate (CER) [13] which corresponds to the proportion of erroneous characters compared to the original text. and the Word Error Rate (WER) [18] which calculates the proportion of erroneous words compared to the total number of words in the original text. A word is considered as erroneous if it contains at least one character error. Table 2 details the OCR error rates at the character and the word levels in the different OCRed version of the three data sets.

\footnotetext{
${ }^{4}$ We have not defined a level combining the LEV-2 degradations because it produces a very poor-quality images and provides unreadable documents.
} 


\begin{tabular}{|l|l|c|c|c|c|c|c|}
\cline { 3 - 8 } \multicolumn{2}{c|}{} & \multicolumn{2}{c|}{ English } & \multicolumn{2}{c|}{ Dutch } & \multicolumn{2}{c|}{ Spanish } \\
\cline { 3 - 8 } \multicolumn{2}{c|}{} & CER & WER & CER & WER & CER & WER \\
\hline \multicolumn{2}{|c|}{ LEV-0 } & 1.7 & 8.5 & 1.6 & 7.8 & 0.7 & 4.8 \\
\hline \multirow{2}{*}{ Bleed-through } & LEV-1 & 1.8 & 8.5 & 1.7 & 8.2 & 0.8 & 4.9 \\
\cline { 2 - 8 } & LEV-2 & 1.8 & 8.6 & 1.8 & 8.9 & 0.8 & 5.4 \\
\hline \multirow{3}{*}{ Blurring } & LEV-1 & 6.3 & 20.0 & 5.9 & 22.0 & 3.0 & 12.0 \\
\cline { 2 - 8 } & LEV-2 & 41.3 & 54.0 & 27.0 & 44.7 & 19.5 & 29.9 \\
\hline \multirow{3}{*}{ Char deg. } & LEV-1 & 3.6 & 21.8 & 4.5 & 25.1 & 2.1 & 14.2 \\
\cline { 2 - 8 } & LEV-2 & 4.3 & 23.7 & 6.4 & 31.6 & 2.7 & 16.3 \\
\hline \multirow{2}{*}{ Phantom deg. } & LEV-1 & 1.7 & 8.8 & 1.6 & 8.0 & 0.8 & 5.5 \\
\cline { 2 - 8 } & LEV-2 & 1.8 & 10.0 & 1.7 & 8.4 & 0.9 & 5.9 \\
\hline \multicolumn{2}{|c|}{ LEV-MIX } & 6.9 & 22.8 & 5.8 & 22.2 & 3.5 & 11.9 \\
\hline
\end{tabular}

Table 2. Estimation of OCR errors rates

As can be seen from Table 2, CER and WER considerably evolve when noises are added to data comparing to re-OCRed clean text (LEV-0). The table shows also that the noise distributed on the documents is homogeneous. The CER is quietly low while the WER is relatively high. Except for Blurring LEV-2 degradation, the CER varies between $\sim 1 \%$ and $\sim 7 \%$ while the error rate at the word level always exceeds $8 \%$ and can reach $\sim 7 \%$ and $\sim 7 \%$ according to the corpus and the type or the level of degradation added. However, errors at the world level are relatively high. OCR error rates show that also that blurring and character degradation are the most critical noise for digitized documents; they generated the highest error rate at both character and word levels.

Despite applying the same degradation through all data, OCR is considerably more accurate through Spanish data, CER and WER rates do not exceed $20 \%$ and $30 \%$. On the other hand OCR error rates, English and Dutch data are more representative, they cover more variable rates that can reach $50 \%$. The bleedthrough and phantom characters added have a slight impact on the effectiveness of the OCR while ink degradation and blurring leads with highest OCR error rates. Among these types of degradation, blurring is the most critical degradation that impacted the OCR outputs.

Concerning NEs, knowing their locations in the original text, we aligned them with corresponding words generated by the OCR. We identified then contaminated NEs and those well recognized by the OCR. A total of 3,623 English named entity tokens have been well recognized by the OCR which represents 63.33\%. This rate achieves $72.14 \%$ for Spanish and $59.87 \%$ for Dutch. All data used in this work are available for public ${ }^{5}$ We provide for each test corpus (English, Dutch and Spanish) the degraded images and their noisy texts extracted

\footnotetext{
${ }^{5}$ www.dropbox.com/s/kw3p162y20s3kiw/Data-TPDL.zip?dl=0. This temporary link is provided solely to reviewers for no further use than the evaluation of this paper's contribution - should the paper be accepted, the data set will be made publicly available on the Zenodo repository.
} 
by the OCR as well as the aligned version with clean data at the word and the character levels.

\section{Evaluation and results}

Obviously, neural networks as well as the training process have several hyperparameters such as character embedding dimension, character-based token embedding, LSTM dimension, token embedding dimension, etc. The same parameters for training and test have been applied on the different dataset : OCRed corpora and clean ones. English embedding has been done using Glove [23] while word2vec [11] is used for Dutch and Spanish words embedding. Table 3 shows results of NER on clean dataset. We used traditional metrics $([\mathrm{P}]$ recision, $[\mathrm{R}]$ ecall and [F1]-score) to evaluate NER systems.

\begin{tabular}{|c|l|c|c|c|c|}
\cline { 3 - 6 } \multicolumn{2}{c|}{} & BLSTM-CRF & BLSTM-CNN & BLSTM-CRF-CNN & CoreNLP \\
\hline \multirow{3}{*}{ English } & $\mathrm{P}$ & 89.54 & 90.57 & 91.05 & 86.35 \\
\cline { 2 - 6 } & $\mathrm{R}$ & 90.81 & 90.98 & 90.75 & 83.88 \\
\cline { 2 - 6 } & $\mathrm{F} 1$ & 90.17 & 90.77 & 90.90 & 85.10 \\
\hline \multirow{3}{*}{ Dutch } & $\mathrm{P}$ & 79.68 & 78.61 & 81.22 & 74.61 \\
\cline { 2 - 6 } & $\mathrm{R}$ & 80.96 & 82.18 & 79.04 & 73.28 \\
\cline { 2 - 6 } & $\mathrm{F} 1$ & 80.31 & 80.36 & 80.12 & 73.94 \\
\hline \multirow{3}{*}{ Spanish } & $\mathrm{P}$ & 87.23 & 87.05 & 87.54 & 75.06 \\
\cline { 2 - 7 } & $\mathrm{R}$ & 83.47 & 83.21 & 83.46 & 76.60 \\
\cline { 2 - 6 } & $\mathrm{F} 1$ & 85.31 & 85.09 & 85.45 & 75.82 \\
\hline
\end{tabular}

Table 3. NER Results on clean data

This first test shows that the results obtained with various methods are globally equivalent for the three languages. We can notice that neural network based approaches propose a bit better results compared to CoreNLP. The same experiment have been run on OCRed dataset. Unsurprisingly, NER accuracy drops proportionally to the rate of OCR errors which is related to the degradation type and level. Table 4 gives F-score of each NER system on noisy data. Results show that comparing to clean data, NER results may loose from 3 to 5 points for LEV-0 OCR-ed data. This proves that OCR has a negative impact for NER task since LEV-0 represents OCR-ed data with no noise added. In other words, even with perfect storage and digitization, NER accuracy may be affected by the OCR quality. For other types of degradation, levels of OCR error rates vary from $8 \%$ to $50 \%$ at the word level and NER F-score may drop from $90 \%$ to $50 \%$ for English. Compared to CoreNLP, deep-learning systems showed a better ability to overcome OCR errors. They achieved satisfactory results when the word error rate is less than $20 \%$. 


\begin{tabular}{|l|c|c|c|c|}
\cline { 2 - 5 } \multicolumn{1}{c|}{ English } & BLSTM-CRF & BLSTM-CNN & BLSTM-CRF-CNN & CoreNLP \\
\hline Clean & 90.17 & 90.77 & $\mathbf{9 0 . 9 0}$ & 85.10 \\
\hline LEV-0 & 86.77 & 86.93 & 87.45 & 79.61 \\
\hline Bleed_LEV-1 & 85.15 & 85.08 & $\mathbf{8 6 . 1 1}$ & 75.72 \\
\hline Bleed_LEV-2 & 84.63 & $\mathbf{8 4 . 7 2}$ & 83.96 & 75.27 \\
\hline Blur_LEV-1 & $\mathbf{7 1 . 0 3}$ & 70.99 & $\mathbf{7 1 . 0 3}$ & 63.39 \\
\hline Blur_LEV-2 & 59.77 & 58.98 & $\mathbf{6 0 . 3 1}$ & 49.15 \\
\hline DegChar_LEV-1 & 73.14 & $\mathbf{7 4 . 2 2}$ & 74.11 & 58.12 \\
\hline DegChar_LEV-2 & $\mathbf{7 0 . 8 5}$ & 69.43 & 68.77 & 55.06 \\
\hline PhantChar_LEV-1 & 85.59 & 85.67 & $\mathbf{8 7 . 0 1}$ & 74.21 \\
\hline PhantChar_LEV-2 & 84.58 & 85.03 & $\mathbf{8 5 . 2 0}$ & 73.66 \\
\hline LEV-MIX & $\mathbf{7 0 . 8 7}$ & 70.11 & 70.82 & 63.35 \\
\hline
\end{tabular}

\begin{tabular}{|l|c|c|c|c|}
\cline { 2 - 5 } \multicolumn{1}{c|}{ Dutch } & BLSTM-CRF & BLSTM-CNN & BLSTM-CRF-CNN & CoreNLP \\
\hline Clean & 80.31 & $\mathbf{8 0 . 6 3}$ & 80.12 & 73.94 \\
\hline LEV-0 & 73.96 & 73.66 & $\mathbf{7 4 . 0 3}$ & 68.36 \\
\hline Bleed_LEV-1 & 72.10 & $\mathbf{7 3 . 4 9}$ & 73.15 & 66.88 \\
\hline Bleed_LEV-2 & 72.06 & $\mathbf{7 2 . 7 5}$ & $\mathbf{7 2 . 7 5}$ & 65.45 \\
\hline Blur_LEV-1 & 63.55 & 63.56 & $\mathbf{6 3 . 7 7}$ & 50.88 \\
\hline Blur_LEV-2 & 42.78 & 42.18 & $\mathbf{4 4 . 5 6}$ & 30.50 \\
\hline DegChar_LEV-1 & 57.42 & $\mathbf{5 7 . 8 9}$ & 56.33 & 47.83 \\
\hline DegChar_LEV-2 & $\mathbf{5 1 . 2 2}$ & 50.98 & 50.78 & 39.16 \\
\hline PhantChar_LEV-1 & 72.23 & $\mathbf{7 3 . 6 6}$ & 73.18 & 67.12 \\
\hline PhantChar_LEV-2 & 70.12 & $\mathbf{7 2 . 9 9}$ & 72.97 & 64.15 \\
\hline LEV-MIX & 64.33 & 64.17 & $\mathbf{6 4 . 8 8}$ & 53.78 \\
\hline
\end{tabular}

\begin{tabular}{|l|c|c|c|c|}
\cline { 2 - 5 } \multicolumn{1}{c|}{ Spanish } & BLSTM-CRF & BLSTM-CNN & BLSTM-CRF-CNN & CoreNLP \\
\hline Clean & 85.31 & 85.09 & $\mathbf{8 5 . 4 5}$ & 75.82 \\
\hline LEV-0 & 85.11 & 84.25 & $\mathbf{8 5 . 1 3}$ & 74.44 \\
\hline Bleed_LEV-1 & $\mathbf{8 4 . 0 8}$ & 83.47 & 84.07 & 70.15 \\
\hline Bleed_LEV-2 & $\mathbf{7 5 . 6 6}$ & 74.99 & 75.12 & 68.77 \\
\hline Blur_LEV-1 & 68.77 & 66.14 & $\mathbf{6 8 . 7 9}$ & 62.41 \\
\hline Blur_LEV-2 & 60.12 & 56.73 & $\mathbf{6 1 . 4 4}$ & 51.32 \\
\hline DegChar_LEV-1 & 64.78 & 63.74 & $\mathbf{6 4 . 9 3}$ & 58.33 \\
\hline DegChar_LEV-2 & 63.01 & 62.09 & $\mathbf{6 4 . 1 2}$ & 52.67 \\
\hline PhantChar_LEV-1 & 77.12 & 74.59 & $\mathbf{7 7 . 2 1}$ & 68.99 \\
\hline PhantChar_LEV-2 & 67.77 & 74.15 & $\mathbf{7 6 . 7 6}$ & 67.37 \\
\hline LEV-MIX & 72.75 & 71.17 & $\mathbf{7 3 . 9 8}$ & 61.14 \\
\hline
\end{tabular}

Table 4. NER F1-score of noisy data

Results in Table 4 indicate also that the best NER F1-score (in bold) can be given by different NER systems according to the type and the level of degradation. For this reason, we calculated the $\delta$ measure which gives the minimum decrease rate between the best F1-score given in clean data and the best F1scores given in noisy data for each type and level of degradation. This measure represents the perfect system that will give the best accuracy for all degrada- 
tion levels. For the three languages, $\delta$ exceeds $40 \%$ in noisy data with WER and CER rates reaching more than 0.4 and 0.5 respectively. Dutch F-score for example decreases under $50 \%$ using any one of the four systems through noisy texts extracted from blurred images with an OCR error rate of $44 \%$ at the word level. Figure 3 shows the evolution of the $\delta$ measure according to degradation. Types of degradation have been sorted according to OCR rates. CER and WER curves are also given for comparative reasons.
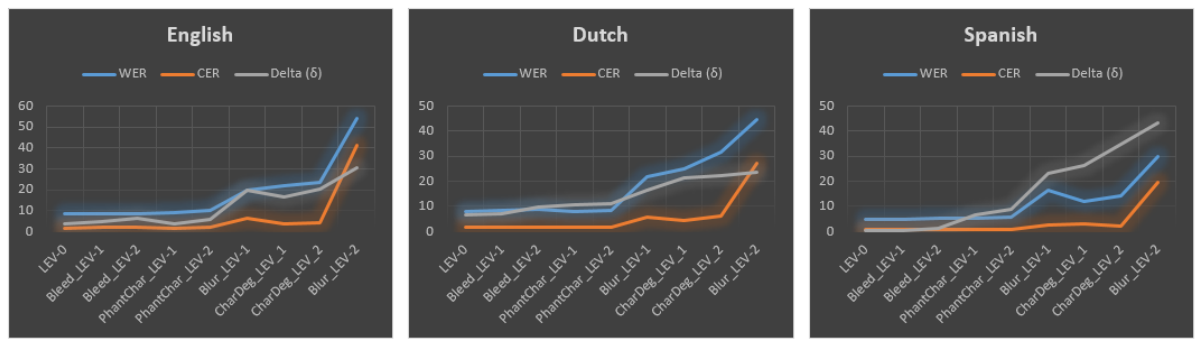

Fig. 3. NER F-score degradation according to OCR error rates

\section{Experiments on historical dataset}

An additional experiment was performed on non synthetic OCRed data, based on Finnish historical newspapers from the National Library of Finland (NLF) [29]. The corpus contains around $450 \mathrm{~K}$ tokens with more than $30 \mathrm{~K}$ NEs. The NLF corpus distinguish only two types of NEs PER and LOC. The CER and WER rates in the OCRed corpus are respectively $6.96 \%$ and $16.67 \%$ which is comparable to error rates given by the simulated bleed_LEV-2 degradation in the CoNLL corpora. Ruokolainen et al. [29] evaluated the NER annotation of the NLF corpus using CoreNLP. The system yielded an overall F1-score of $72 \%$ over OCRed texts which presents a loss of around 10 points compared to clean texts. This decrease is mostly equivalent to those obtained on the OCRed synthetic data using CoreNLP (cf. Table 4). With the same OCR error rates NER F1-score on the English corpus presents a loss of $9.83 \%$ compared to results on clean corpus.

Using BLSTM-CRF, NER F1-score achieves $89.8 \%$ and $87.4 \%$ on clean and OCRed data respectively which represents a decrease rate of 2.4 percentage points. The corresponding rates in the CoNLL corpora are between 4 and 8 percentage points as shown in Figure 3. Finnish results are slightly better than those obtained with synthetic data using BLSTM-CRF. This is not unexpected since the Finnish training set is larger than the CoNLL data sets. In addition the set of NEs in the NLF corpus is less refined than the set used in the CoNLL corpora. As we showed in Table 4, neural network based systems outperforms CoreNLP, we have thus reported the same experiment on the NLF corpus using BLSTM-CRF. Results are shown in table 5. 


\begin{tabular}{|c|l|c|c|c|}
\cline { 3 - 5 } \multicolumn{2}{c|}{} & PER & LOC & TOT \\
\hline \multirow{3}{*}{ clean } & P & $93.39 \%$ & $87.43 \%$ & $90.82 \%$ \\
\cline { 2 - 5 } & R & $91.86 \%$ & $84.68 \%$ & $88.74 \%$ \\
\cline { 2 - 5 } & F1 & $92.62 \%$ & $86.03 \%$ & $89.77 \%$ \\
\hline \multirow{3}{*}{ OCRed } & $\mathrm{P}$ & $89.68 \%$ & $83.31 \%$ & $86.97 \%$ \\
\cline { 2 - 5 } & $\mathrm{R}$ & $91.06 \%$ & $83.54 \%$ & $87.83 \%$ \\
\cline { 2 - 5 } & F1 & $90.36 \%$ & $83.42 \%$ & $87.40 \%$ \\
\hline
\end{tabular}

Table 5. Results on the NLF corpus

Results using the neural-network system are largely outperforming CoreNLP performances. For clean data, we obtained an overall F1-score of $89 \%$ (to be compared to $82 \%$ ). More importantly, for OCRed data, the NER F1-score reaches $90.4 \%$ for PER and $83.4 \%$ for LOC, resulting in an improvement of 11 points for both types of NEs. Despite the complexity of the NER task and the occurrence of several types of errors in the documents of digital libraries, the systems achieved interesting results. This proves that they can be used to distinguish ambiguous named entities in degraded documents. Some word correction strategies, such as auto-encoders, language models, and so on, could be used to decrease the impact of OCR degradation on NER.

\section{Conclusion}

This paper is the most systematic evaluation of the impact of OCR errors on NER systems over multilingual datasets. We evaluated four machine-learning systems over three available data sets in English, Dutch and Spanish. We reOCRed these collections and added four types of noises at two different levels in order to simulate various OCR output. All the noisy texts have been aligned with their corresponding ground truth in order to test the NER system through noisy data and to observe the evolution of their accuracy. This new data set was made publicly available to the community. Such resources combined OCRed data aligned with their clean version are very useful for two reasons. First they can be used to train NLP algorithms over collections of documents that have been through an OCR process, as is notably the case of historical documents. Second, they can be used to estimate the impact of OCR over NLP applications and lead to recommendations, for instance on what application can reasonably be run over a document collection given its OCR quality.

We have studied the correlation between OCR error rates and NER accuracy using four effective systems. We showed that NER accuracy drops from $90 \%$ to $50 \%$ when the word error rate increase from $8 \%$ to $50 \%$. These experiments were validated on a real OCR dataset in Finnish, where our systematic study allowed to outperform the best-known results by 11.4 percentage points.

This work showed that specific post OCR correction should be developed in order to improve NER results, and so information access by end users. For instance, we are convinced that usage of knowledge (frequencies of errors on NEs) would bring a substantial gain of NER systems. 


\section{References}

1. Bikel, D.M., Schwartz, R., Weischedel, R.M.: An algorithm that learns what's in a name. Machine learning 34(1-3), 211-231 (1999)

2. Chiron, G., Doucet, A., Coustaty, M., Visani, M., Moreux, J.P.: Impact of ocr errors on the use of digital libraries: towards a better access to information. In: Proceedings of the 17th ACM/IEEE Joint Conference on Digital Libraries. pp. 249-252. IEEE Press (2017)

3. Chiu, J.P., Nichols, E.: Named entity recognition with bidirectional lstm-cnns. arXiv preprint arXiv:1511.08308 (2015)

4. Collobert, R., Weston, J., Bottou, L., Karlen, M., Kavukcuoglu, K., Kuksa, P.: Natural language processing (almost) from scratch. Journal of Machine Learning Research 12(Aug), 2493-2537 (2011)

5. Croft, W., Harding, S., Taghva, K., Borsack, J.: An evaluation of information retrieval accuracy with simulated ocr output. In: Symposium on Document Analysis and Information Retrieval. pp. 115-126 (1994)

6. F, E., Sang, T.K.: Introduction to the conll-2002 shared task: Languageindependent named entity recognition. In: Proceedings of CoNLL-2002. pp. 155$158(2002)$

7. Favre, B., Béchet, F., Nocéra, P.: Robust named entity extraction from large spoken archives. In: Proceedings of the conference on Human Language Technology and Empirical Methods in Natural Language Processing. pp. 491-498. Association for Computational Linguistics (2005)

8. Finkel, J.R., Grenager, T., Manning, C.: Incorporating non-local information into information extraction systems by gibbs sampling. In: Proceedings of the 43rd annual meeting on association for computational linguistics. pp. 363-370. Association for Computational Linguistics (2005)

9. Gali, K., Surana, H., Vaidya, A., Shishtla, P.M., Sharma, D.M.: Aggregating machine learning and rule based heuristics for named entity recognition. In: Proceedings of the IJCNLP-08 Workshop on Named Entity Recognition for South and South East Asian Languages (2008)

10. Gefen, A.: Les enjeux épistémologiques des humanités numériques. Socio-La nouvelle revue des sciences sociales (4), 61-74 (2014)

11. Goldberg, Y., Levy, O.: word2vec explained: deriving mikolov et al.'s negativesampling word-embedding method. arXiv preprint arXiv:1402.3722 (2014)

12. Grishman, R., Sundheim, B.: Message understanding conference-6: A brief history. In: COLING 1996 Volume 1: The 16th International Conference on Computational Linguistics. vol. 1 (1996)

13. Holley, R.: How good can it get? analysing and improving ocr accuracy in large scale historic newspaper digitisation programs. D-Lib Magazine 15(3/4) (2009)

14. Jing, H., Lopresti, D., Shih, C.: Summarizing noisy documents. In: Proceedings of the Symposium on Document Image Understanding Technology. pp. 111-119 (2003)

15. Journet, N., Visani, M., Mansencal, B., Van-Cuong, K., Billy, A.: Doccreator: A new software for creating synthetic ground-truthed document images. Journal of imaging 3(4), 62 (2017)

16. Lample, G., Ballesteros, M., Subramanian, S., Kawakami, K., Dyer, C.: Neural architectures for named entity recognition. arXiv preprint arXiv:1603.01360 (2016)

17. Lopresti, D.: Optical character recognition errors and their effects on natural language processing. International Journal on Document Analysis and Recognition (IJDAR) 12(3), 141-151 (2009) 
18. Lund, W.B., Kennard, D.J., Ringger, E.K.: Combining multiple thresholding binarization values to improve ocr output. In: Document Recognition and Retrieval XX. vol. 8658, p. 86580R. International Society for Optics and Photonics (2013)

19. Ma, X., Hovy, E.: End-to-end sequence labeling via bi-directional lstm-cnns-crf. arXiv preprint arXiv:1603.01354 (2016)

20. Miller, D., Boisen, S., Schwartz, R., Stone, R., Weischedel, R.: Named entity extraction from noisy input: speech and ocr. In: Proceedings of the sixth conference on Applied natural language processing. pp. 316-324. Association for Computational Linguistics (2000)

21. Nadeau, D., Sekine, S.: A survey of named entity recognition and classification. Lingvisticae Investigationes 30(1), 3-26 (2007)

22. Palmer, D.D., Ostendorf, M.: Improving information extraction by modeling errors in speech recognizer output. In: Proceedings of the first international conference on Human language technology research. pp. 1-5. Association for Computational Linguistics (2001)

23. Pennington, J., Socher, R., Manning, C.: Glove: Global vectors for word representation. In: Proceedings of the 2014 conference on empirical methods in natural language processing (EMNLP). pp. 1532-1543 (2014)

24. Peters, M.E., Neumann, M., Iyyer, M., Gardner, M., Clark, C., Lee, K., Zettlemoyer, L.: Deep contextualized word representations. arXiv preprint arXiv:1802.05365 (2018)

25. Ramshaw, L.A., Marcus, M.P.: Text chunking using transformation-based learning. In: Natural language processing using very large corpora, pp. 157-176. Springer (1999)

26. Riedl, M., Padó, S.: A named entity recognition shootout for German. In: Proceedings of ACL. pp. 120-125. Melbourne, Australia (2018), http://aclweb.org/ anthology/P18-2020.pdf

27. Ritter, A., Clark, S., Etzioni, O., et al.: Named entity recognition in tweets: an experimental study. In: Proceedings of the conference on empirical methods in natural language processing. pp. 1524-1534. Association for Computational Linguistics (2011)

28. Rodriquez, K.J., Bryant, M., Blanke, T., Luszczynska, M.: Comparison of named entity recognition tools for raw ocr text. In: KONVENS. pp. 410-414 (2012)

29. Ruokolainen, T., Kettunen, K.: À la recherche du nom perdu-searching for named entities with stanford ner in a finnish historical newspaper and journal collection. In: 13th IAPR International Workshop on Document Analysis Systems (2018)

30. Taghva, K., Borsack, J., Condit, A.: Effects of ocr errors on ranking and feedback using the vector space model. Inf. Process. Manage. 32(3), 317-327 (1996)

31. Tjong Kim Sang, E.F., De Meulder, F.: Introduction to the conll-2003 shared task: Language-independent named entity recognition. In: Proceedings of the seventh conference on Natural language learning at HLT-NAACL 2003-Volume 4. pp. 142 147. Association for Computational Linguistics (2003)

32. Yalniz, I.Z., Manmatha, R.: A fast alignment scheme for automatic ocr evaluation of books. In: Document Analysis and Recognition (ICDAR), 2011 International Conference on. pp. 754-758. IEEE (2011)

33. Yaser, A.O.: Effect of degraded input on statistical machine translation. In: 2005 Symposium on Document Image Understanding Technology. p. 103 (2005)

34. Zuccon, G., Nguyen, A.N., Bergheim, A., Wickman, S., Grayson, N.: The impact of ocr accuracy on automated cancer classification of pathology reports. In: HIC. pp. 250-256 (2012) 